\title{
A New Tower Over Cubic Finite Fields
}

\author{
Alp Bassa*, Arnaldo Garcia ${ }^{\dagger \dagger}$ and Henning Stichtenoth*
}

We present a new explicit tower of function fields $\left(F_{n}\right)_{n \geq 0}$ over the finite field with $\ell=q^{3}$ elements, where the limit of the ratios (number of rational places of $\left.F_{n}\right) /\left(\right.$ genus of $F_{n}$ ) is bigger or equal to $2\left(q^{2}-1\right) /(q+2)$. This tower contains as a subtower the tower which was introduced by BezerraGarcia-Stichtenoth (see [3]), and in the particular case $q=2$ it coincides with the tower of van der Geer-van der Vlugt (see [12]). Many features of the new tower are very similar to those of the optimal wild tower in [8] over the quadratic field $\mathbb{F}_{q^{2}}$ (whose modularity was shown in [6] by Elkies).

\section{Introduction}

Let $F / \mathbb{F}_{\ell}$ be an algebraic function field of one variable whose full constant field is the finite field $\mathbb{F}_{\ell}$ of cardinality $\ell$. We denote by $g(F)$ the genus and by $N(F)$ the number of rational places (i.e., places of degree one) of $F / \mathbb{F}_{\ell}$. The classical Hasse-Weil Theorem states that $N(F) \leq \ell+1+2 g(F) \sqrt{\ell}$.

Ihara [13] was the first to observe that this inequality can be improved substantially if the genus of $F$ is large with respect to $\ell$. He introduced the real number

$$
A(\ell):=\limsup _{g(F) \rightarrow \infty} \frac{N(F)}{g(F)}
$$

where $F$ runs over all function fields over $\mathbb{F}_{\ell}$. This number $A(\ell)$ is of fundamental importance to the theory of function fields over a finite field, since it gives information about how many rational places a function field $F / \mathbb{F}_{\ell}$ of large genus can have. While the Hasse-Weil Theorem gives that $A(\ell) \leq 2 \sqrt{\ell}$, Ihara showed that $A(\ell) \leq \sqrt{2 \ell}$ for any $\ell$ and that $A(\ell) \geq \sqrt{\ell}-1$ for $\ell$ a square. Later Drinfel'd and Vlădut [4] showed that

$$
A(\ell) \leq \sqrt{\ell}-1 \quad \text { for any } \ell .
$$

\footnotetext{
*Sabancı University, MDBF, Orhanlı, 34956 Tuzla, İstanbul, Turkey

${ }^{\dagger}$ Instituto Nacional de Matemática Pura e Aplicada, IMPA, Estrada Dona Castorina 110, 22460-320, Rio de Janeiro, RJ, Brazil

${ }^{\ddagger}$ A. Garcia was partially supported by PRONEX-FAPERJ and CNPq-Brazil (Proc. 307569/2006-3), and also by Sabancı University, İstanbul
} 
Hence we have the equality $A(\ell)=\sqrt{\ell}-1$ for $\ell$ a square (see also [5], [7], [17]).

Much less is known if $\ell$ is not a square. One knows that for any $\ell$ (see Serre [15])

$$
A(\ell) \geq c \cdot \log \ell, \quad \text { for some constant } c>0 .
$$

For $\ell=p^{3}$ ( $p$ a prime number), the best known lower bound for $A(\ell)$ is due to Zink [18]:

$$
A\left(p^{3}\right) \geq \frac{2\left(p^{2}-1\right)}{p+2} .
$$

Zink obtained this result using degenerations of Shimura modular surfaces. Zink's bound was generalized by Bezerra, Garcia and Stichtenoth [3] who showed that

$$
A\left(q^{3}\right) \geq \frac{2\left(q^{2}-1\right)}{q+2}
$$

holds for all prime powers $q$. For more information and references concerning Ihara's quantity $A(\ell)$ we refer to the recent survey article [11].

In order to obtain lower bounds for $A(\ell)$, it is natural to study towers of function fields; i.e., one considers sequences $\mathcal{G}=\left(G_{0}, G_{1}, G_{2}, \ldots\right)$ of function fields $G_{i}$ over $\mathbb{F}_{\ell}$ with $G_{0} \subseteq G_{1} \subseteq G_{2} \subseteq \ldots$ such that $g\left(G_{i}\right) \rightarrow \infty$. It is easy to see that the limit

$$
\lambda(\mathcal{G}):=\lim _{i \rightarrow \infty} \frac{N\left(G_{i}\right)}{g\left(G_{i}\right)}
$$

always exists (see [8]), and it is clear that $0 \leq \lambda(\mathcal{G}) \leq A(\ell)$.

A particularly interesting example is the tower $\mathcal{H}=\left(H_{0}, H_{1}, H_{2}, \ldots\right)$ over the field $\mathbb{F}_{\ell}$ with $\ell=q^{2}$, which is defined recursively as follows (see [8]): $H_{0}=\mathbb{F}_{\ell}\left(u_{0}\right)$ is the rational function field, and for all $i \geq 0$ one considers the field $H_{i+1}=H_{i}\left(u_{i+1}\right)$ with

$$
u_{i+1}^{q}+u_{i+1}=\frac{u_{i}^{q}}{u_{i}^{q-1}+1} .
$$

This tower over $\mathbb{F}_{q^{2}}$ has the limit $\lambda(\mathcal{H})=q-1=\sqrt{\ell}-1$, and therefore it attains the Drinfel'd-Vlădut bound (1). Elkies [6] has shown that $\mathcal{H}$ is in fact a modular tower.

In [3] the following tower $\mathcal{E}=\left(E_{0}, E_{1}, E_{2}, \ldots\right)$ over a cubic field $\mathbb{F}_{\ell}$ with $\ell=q^{3}$ is considered: again $E_{0}=\mathbb{F}_{\ell}\left(v_{0}\right)$ is the rational function field, and for $i \geq 0$ one considers the field $E_{i+1}=E_{i}\left(v_{i+1}\right)$ with

$$
\frac{1-v_{i+1}}{v_{i+1}^{q}}=\frac{v_{i}^{q}+v_{i}-1}{v_{i}}
$$

The limit $\lambda(\mathcal{E})$ satisfies the inequality (thus proving Inequality (3)):

$$
\lambda(\mathcal{E}) \geq \frac{2\left(q^{2}-1\right)}{q+2}
$$


The tower $\mathcal{H}$ over the quadratic field $\mathbb{F}_{\ell}$ with $\ell=q^{2}$ which is defined by Eqn. (4) has some nice features which allow a rather simple proof of the equality $\lambda(\mathcal{H})=q-1$, see [9]. The most important one is that all extensions $H_{i+1} / H_{i}$ are Galois of degree $q$, and for all places $Q \mid P$ with ramification index $e=e(Q \mid P)>1$ in $H_{i+1} / H_{i}$, the different exponent is $d(Q \mid P)=2(e-1)$.

In contrast, the tower $\mathcal{E}$ over the cubic field $\mathbb{F}_{\ell}$ with $\ell=q^{3}$ which is defined by Eqn. (5) is much more complicated. Here (for $q \neq 2$ ) the extensions $E_{i+1} / E_{i}$ are not even Galois, and there occurs tame and also wild ramification in $E_{i+1} / E_{i}$. The determination of the genus of $E_{n}$ in [3] requires long and rather technical calculations. In [1] these calculations were replaced by a structural argument, thus obtaining a simpler proof of Inequality (6) without the explicit determination of $g\left(E_{n}\right)$. In [14], Ihara provides a construction of an infinite Galois extension, which contains the tower $\mathcal{E}$ and exhibits the splitting places of $\mathcal{E}$ in a more natural way. He also introduces a higher order differential which is invariant under the action of the associated infinite Galois group.

In this paper we present a new tower $\mathcal{F}$ over the cubic field $\mathbb{F}_{\ell}$ with $\ell=q^{3}$, whose limit also satisfies the inequality $\lambda(\mathcal{F}) \geq 2\left(q^{2}-1\right) /(q+2)$ and which has nicer properties than the tower given by the recursion in Eqn. (5). This new tower $\mathcal{F}=\left(F_{0}, F_{1}, F_{2}, \ldots\right)$ over $\mathbb{F}_{\ell}$ is defined as follows: $F_{0}=\mathbb{F}_{\ell}\left(x_{0}\right)$ is the rational function field over $\mathbb{F}_{\ell}$, and for $n \geq 0$ one sets $F_{n+1}=F_{n}\left(x_{n+1}\right)$ with

$$
\left(x_{n+1}^{q}-x_{n+1}\right)^{q-1}+1=\frac{-x_{n}^{q(q-1)}}{\left(x_{n}^{q-1}-1\right)^{q-1}} .
$$

We would like to point out that our proof, that the limit of this new tower also satisfies the inequality $\lambda(\mathcal{F}) \geq 2\left(q^{2}-1\right) /(q+2)$, is much easier, shorter and less computational than the proofs in [3] and [1] for the tower $\mathcal{E}$. Moreover, since we show that $\mathcal{E}$ is a subtower of $\mathcal{F}$ we also get a new and simpler proof of Inequality (6); in fact, it follows from $[8]$ that $\lambda(\mathcal{E}) \geq \lambda(\mathcal{F})$ when $\mathcal{E}$ is a subtower of $\mathcal{F}$.

Another remark is that while for the two towers over $\mathbb{F}_{q^{2}}$ presented in [7] and [8] the subtower (i.e., the tower $\mathcal{H}$ in [8]) was easier to handle, for the two towers $\mathcal{E}$ and $\mathcal{F}$ over $\mathbb{F}_{q^{3}}$ the supertower (i.e., the tower $\mathcal{F}$ ) turns out to be much easier to handle.

Finally we note that the tower $\mathcal{F}$ coincides with the van der Geer-van der Vlugt tower in [12] when $q=2$, and also that the towers $\mathcal{F}$ and $\mathcal{H}$ have surprising similarities (see Section 8).

This paper is organized as follows: In Sec. 2 we introduce the sequence of function fields $F_{0}, F_{1}, F_{2}, \ldots$ over a field $K \supseteq \mathbb{F}_{q}$ recursively given by Eqn. (7) and we show in Theorem 2.2 that they define a tower $\mathcal{F}$ over $K$ (i.e., $F_{0} \subsetneq F_{1} \subsetneq F_{2} \subsetneq \ldots$, and $K$ is the full constant field of all fields $F_{n}$ ). In Sec. 3 it is shown that for $K=\mathbb{F}_{q^{3}}$ there exist $q^{3}-q$ rational places of $F_{0}$ which split completely in all extensions $F_{n} / F_{0}$, thus providing many rational places of the function fields $F_{n} / \mathbb{F}_{q^{3}}$. In Sec. 4 and Sec. 5 we study ramification in the first steps $F_{0} \subseteq F_{1} \subseteq F_{2}$ of the tower. We note that the methods in Sec. 4 and Sec. 5 involve just simple calculations about ramification in certain Galois extensions $K(x) / K(w)$ of rational function fields. Section 6 is the core of this paper. The results from Sec. 4 and Sec. 5 are used in Sec. 6 to give an upper bound for the genus of the 
$n$-th function field $F_{n}$ of the tower (see Thm. 6.5). The main tool here is a variant of Abhyankar's Lemma (see Lemma 6.2) dealing with ramification in composites of certain wildly ramified extensions. Putting together the results from Sec. 3 and Sec. 6 we obtain in Sec. 7 the inequality $\lambda(\mathcal{F}) \geq 2\left(q^{2}-1\right) /(q+2)$ for $K=\mathbb{F}_{q^{3}}$, which is the main result of the paper. Finally, in Sec. 8 we point out some surprising analogies between the tower $\mathcal{F}$ over $\mathbb{F}_{q^{3}}$ and the tower $\mathcal{H}$ over $\mathbb{F}_{q^{2}}$ which is defined by Eqn. (4). We also show that the above-mentioned tower $\mathcal{E}$ is a subtower of $\mathcal{F}$.

NOTATIONS: We consider function fields $F / K$ where $K$ is the full constant field of $F$. In most cases $K$ will be a finite field or the algebraic closure $\overline{\mathbb{F}}_{q}$ of a finite field. We denote by $\mathbb{P}(F)$ the set of places of $F / K$. For $P \in \mathbb{P}(F)$, we will denote by $v_{P}$ the corresponding discrete valuation of $F / K$ and by $\mathcal{O}_{P}$ the valuation ring of $P$. For $z \in \mathcal{O}_{P}$ we denote by $z(P)$ the residue class of $z$ in $\mathcal{O}_{P} / P$. We denote by $\operatorname{deg}(P)$ the degree of $P$. In particular, if $P$ is a place of degree one, then $z(P) \in K$.

For a finite separable extension $E$ of $F$ and a place $Q \in \mathbb{P}(E)$ we will denote by $\left.Q\right|_{F}$ the restriction of $Q$ to $F$. We write $Q \mid P$ if the place $Q \in \mathbb{P}(E)$ lies over the place $P \in \mathbb{P}(F)$. In this situation, we denote by $e(Q \mid P)$ and $d(Q \mid P)$ the ramification index and the different exponent of $Q \mid P$, respectively. The place $P \in \mathbb{P}(F)$ is said to be totally ramified in $E / F$ if there is a place $Q \in \mathbb{P}(E)$ above $P$ with $e(Q \mid P)=[E: F]$. It is said to be completely splitting in $E / F$ if there are $n=[E: F]$ distinct places of $E$ above $P$.

Let $E / F$ be a Galois extension of function fields, let $P \in \mathbb{P}(F)$ and $Q \in \mathbb{P}(E)$ above the place $P$. We say that $Q \mid P$ is weakly ramified if the second ramification group $G_{2}(Q \mid P)=1$; in other words, if $e(Q \mid P)=e_{0} \cdot e_{1}$ where $\left(e_{0}, p\right)=1$ and $e_{1}=p^{j}$ is a power of the characteristic $p$ of $F$, then $d(Q \mid P)=\left(e_{0} e_{1}-1\right)+\left(e_{1}-1\right)$.

If $F=K(x)$ is a rational function field, we will write $(x=\alpha)$ for the place of $F$ which is the zero of $x-\alpha$ (where $\alpha \in K$ ), and $(x=\infty$ ) for the pole of $x$ in $K(x) / K$.

\section{The tower}

Let $K$ be a field of characteristic $p>0$, let $q$ be a power of $p$ and assume that $\mathbb{F}_{q} \subseteq K$. We study the sequence $\mathcal{F}=\left(F_{0}, F_{1}, F_{2}, \ldots\right)$ of function fields $F_{i} / K$ which is defined recursively as follows: $F_{0}=K\left(x_{0}\right)$ is the rational function field, and for $n \geq 0$ let $F_{n+1}=F_{n}\left(x_{n+1}\right)$ where $x_{n+1}$ satisfies the equation over $F_{n}$ below:

$$
\left(x_{n+1}^{q}-x_{n+1}\right)^{q-1}+1=\frac{-x_{n}^{q(q-1)}}{\left(x_{n}^{q-1}-1\right)^{q-1}} .
$$

Remark 2.1. We set

$$
f(T):=\left(T^{q}-T\right)^{q-1}+1 \in K[T] .
$$

Then Eqn. (8) can be written as

$$
f\left(x_{n+1}\right)=\frac{1}{1-f\left(1 / x_{n}\right)} .
$$


We also remark that $f(T)=\left(T^{q^{2}}-T\right) /\left(T^{q}-T\right)$, hence the roots of $f(T)$ are exactly the elements $\beta \in \mathbb{F}_{q^{2}} \backslash \mathbb{F}_{q}$. This property of the polynomial $f(T)$ will play an important role in Sections 3 and 4 .

Theorem 2.2. Let $\mathcal{F}$ be the sequence of function fields $F_{n}$ over $K$ which is defined by Eqn. (8). Then $\mathcal{F}$ is a tower over $K$, and more precisely the following hold:

(i) The extensions $F_{n+1} / F_{n}$ are Galois for all $n \geq 0$.

(ii) $\left[F_{1}: F_{0}\right]=q(q-1)$ and $\left[F_{n+1}: F_{n}\right]=q$ for all $n \geq 1$.

(iii) $K$ is the full constant field of $F_{n}$, for all $n \geq 0$.

The proof of Thm. 2.2 is given in several steps.

Lemma 2.3. $F_{n+1} / F_{n}$ is Galois and $\left[F_{n+1}: F_{n}\right]$ divides $q(q-1)$, for all $n \geq 0$.

Proof. We set

$$
u_{n}:=\frac{-x_{n}^{q(q-1)}}{\left(x_{n}^{q-1}-1\right)^{q-1}} .
$$

Then $x_{n+1}$ is a root of the polynomial $f_{n}(T):=\left(T^{q}-T\right)^{q-1}+1-u_{n} \in F_{n}[T]$. The other roots of $f_{n}(T)$ are the elements $a x_{n+1}+b$ with $a \in \mathbb{F}_{q}^{\times}$and $b \in \mathbb{F}_{q}$. Therefore $F_{n+1}$ is the splitting field of $f_{n}(T)$ over $F_{n}$ and the extension $F_{n+1} / F_{n}$ is Galois.

Let $G_{n+1}$ be the Galois group of $F_{n+1} / F_{n}$. Every element $\sigma \in G_{n+1}$ acts on the function $x_{n+1}$ as $\sigma\left(x_{n+1}\right)=a_{\sigma} x_{n+1}+b_{\sigma}$, and the map

$$
\sigma \mapsto\left(\begin{array}{cc}
a_{\sigma} & 0 \\
b_{\sigma} & 1
\end{array}\right)
$$

is a monomorphism of $G_{n+1}$ into the group of invertible $2 \times 2$-matrices over $\mathbb{F}_{q}$ of the form $\left(\begin{array}{cc}a & 0 \\ b & 1\end{array}\right)$. This group has order $q(q-1)$, and hence $\operatorname{ord}\left(G_{n+1}\right)$ divides $q(q-1)$.

Lemma 2.4. Let $P_{0}=\left(x_{0}=\infty\right)$ be the pole of $x_{0}$ in $F_{0}$ and let $P_{n}$ be a place of $F_{n}$ above $P_{0}$. For $i=1, \ldots, n$ we set $P_{i}:=\left.P_{n}\right|_{F_{i}}$ and $e^{(i)}:=e\left(P_{i} \mid P_{i-1}\right)$. Then the place $P_{i}$ is a pole of $x_{i}$. Moreover, $v_{P_{i}}\left(x_{i}\right)$ divides $(q-1)^{i}$, and $e^{(i)} \equiv 0 \bmod q$, for $1 \leq i \leq n$.

Proof. Let $u_{i} \in F_{i}$ be defined as in Eqn. (11). We prove the lemma by induction. For the case $i=1$, we have $v_{P_{1}}\left(u_{0}\right)=e^{(1)} \cdot v_{P_{0}}\left(u_{0}\right)=-e^{(1)} \cdot(q-1)$. From the equation $\left(x_{1}^{q}-x_{1}\right)^{q-1}+1=u_{0}$, it follows that $v_{P_{1}}\left(x_{1}\right)<0$ and therefore

$$
v_{P_{1}}\left(\left(x_{1}^{q}-x_{1}\right)^{q-1}+1\right)=q \cdot(q-1) \cdot v_{P_{1}}\left(x_{1}\right) .
$$

We conclude that $q \cdot v_{P_{1}}\left(x_{1}\right)=-e^{(1)}$. To finish this case, notice that $e^{(1)}$ divides the degree $\left[F_{1}: F_{0}\right]$, and $\left[F_{1}: F_{0}\right]$ divides $q(q-1)$ (by Lemma 2.3). Hence it follows that $v_{P_{1}}\left(x_{1}\right)$ divides $(q-1)$ and that $e^{(1)} \equiv 0 \bmod q$. 
Now we assume that $v_{P_{i}}\left(x_{i}\right)<0$ and $v_{P_{i}}\left(x_{i}\right)$ divides $(q-1)^{i}$ for some $i \in\{1, \ldots, n-1\}$. From Eqn. (11) we obtain $v_{P_{i}}\left(u_{i}\right)=(q-1) \cdot v_{P_{i}}\left(x_{i}\right)$, hence

$$
v_{P_{i+1}}\left(u_{i}\right)=e^{(i+1)} \cdot(q-1) \cdot v_{P_{i}}\left(x_{i}\right)<0 .
$$

Since $\left(x_{i+1}^{q}-x_{i+1}\right)^{q-1}+1=u_{i}$, it follows that $P_{i+1}$ is a pole of $x_{i+1}$ and

$$
q(q-1) \cdot v_{P_{i+1}}\left(x_{i+1}\right)=e^{(i+1)} \cdot(q-1) \cdot v_{P_{i}}\left(x_{i}\right) .
$$

Now we finish as in the case $i=1$; we conclude that $e^{(i+1)} \equiv 0 \bmod q$ and that $v_{P_{i+1}}\left(x_{i+1}\right)$ divides $(q-1)^{i+1}$.

Lemma 2.5. $\left[F_{n+1}: F_{n}\right] \equiv 0 \bmod q$ for all $n \geq 0$.

Proof. Follows directly from Lemmas 2.3 and 2.4.

Lemma 2.6. $\left[F_{1}: F_{0}\right]=q(q-1)$, and $K$ is the full constant field of $F_{1}$.

Proof. By definition, $F_{1}=K\left(x_{0}, x_{1}\right)$ with

$$
\left(x_{1}^{q}-x_{1}\right)^{q-1}+1=\frac{-x_{0}^{q(q-1)}}{\left(x_{0}^{q-1}-1\right)^{q-1}}=u_{0} .
$$

It follows that

$$
\left[K\left(x_{0}\right): K\left(u_{0}\right)\right]=\left[K\left(x_{1}\right): K\left(u_{0}\right)\right]=q(q-1) .
$$

From Eqn. (12) it is obvious that the place $\left(u_{0}=0\right)$ of $K\left(u_{0}\right)$ is totally ramified in the extension $K\left(x_{0}\right) / K\left(u_{0}\right)$. The place of $K\left(x_{0}\right)$ above $\left(u_{0}=0\right)$ is the place $\left(x_{0}=0\right)$, and we have $e\left(\left(x_{0}=0\right) \mid\left(u_{0}=0\right)\right)=q(q-1)$.

However, in the extension $K\left(x_{1}\right) / K\left(u_{0}\right)$ the place $\left(u_{0}=0\right)$ is unramified, since the polynomial $\left(x_{1}^{q}-x_{1}\right)^{q-1}+1$ does not have multiple roots. Let $Q$ be a place of $K\left(x_{1}\right)$ lying above $\left(u_{0}=0\right)$ and let $R$ be a place of $K\left(x_{0}, x_{1}\right)$ above $Q$. It follows from above that $e(R \mid Q)=q(q-1)$. Therefore $\left[K\left(x_{0}, x_{1}\right): K\left(x_{1}\right)\right]=q(q-1)$, and $K$ is algebraically closed in $K\left(x_{0}, x_{1}\right)=F_{1}$ (as there is a place which is totally ramified in $F_{1} / K\left(x_{1}\right)$ ). The assertion $\left[F_{1}: F_{0}\right]=q(q-1)$ follows since $\left[F_{1}: F_{0}\right]=\left[F_{1}: K\left(x_{1}\right)\right]$ by Eqn. (13).

The next lemma shows a striking property of the recursion in Eqn. (8) for $n \geq 1$. It gives a simple Artin-Schreier equation for the extension $F_{n+1} / F_{n}$ of degree $q$.

Lemma 2.7. For each $n \geq 1$ there is some $\mu \in \mathbb{F}_{q}^{\times}$such that

$$
x_{n+1}^{q}-x_{n+1}=\mu \cdot \frac{x_{n-1}^{q}}{\left(x_{n-1}^{q-1}-1\right) \cdot\left(x_{n}^{q-1}-1\right)} .
$$

Proof. By Eqn. (8) we have

$$
\left(x_{n+1}^{q}-x_{n+1}\right)^{q-1}+1=\frac{-x_{n}^{q(q-1)}}{\left(x_{n}^{q-1}-1\right)^{q-1}} \text { and }\left(x_{n}^{q}-x_{n}\right)^{q-1}+1=\frac{-x_{n-1}^{q(q-1)}}{\left(x_{n-1}^{q-1}-1\right)^{q-1}} .
$$


Hence we get

$$
\begin{aligned}
\left(x_{n+1}^{q}-x_{n+1}\right)^{q-1} & =\frac{-x_{n}^{q(q-1)}}{\left(x_{n}^{q-1}-1\right)^{q-1}}-1=\frac{-\left(\left(x_{n}^{q}-x_{n}\right)^{q-1}+1\right)}{\left(x_{n}^{q-1}-1\right)^{q-1}} \\
& =\frac{x_{n-1}^{q(q-1)}}{\left(x_{n-1}^{q-1}-1\right)^{q-1} \cdot\left(x_{n}^{q-1}-1\right)^{q-1}}=\left(\frac{x_{n-1}^{q}}{\left(x_{n-1}^{q-1}-1\right) \cdot\left(x_{n}^{q-1}-1\right)}\right)^{q-1} .
\end{aligned}
$$

Proof of Theorem 2.2. Putting together the results of the lemmas above, one gets the assertions of Thm. 2.2.

\section{Splitting places in the tower over $K=\mathbb{F}_{\ell}$ for $\ell=q^{3}$}

In this section we consider the tower $\mathcal{F}=\left(F_{0}, F_{1}, F_{2}, \ldots\right)$ which was introduced in Sec. 2, over the field $K=\mathbb{F}_{\ell}$ with $\ell=q^{3}$. We will show that many rational places of the field $F_{0}=\mathbb{F}_{\ell}\left(x_{0}\right)$ split completely in $\mathcal{F}$; i.e., they split completely in all extensions $F_{n} / F_{0}$. This means that the function fields $F_{n} / \mathbb{F}_{\ell}$ have "many" rational places. As in Sec. 2, let

$$
f(T)=\left(T^{q}-T\right)^{q-1}+1 \in \mathbb{F}_{q}[T] .
$$

For $q=2$ we have obviously that $f(T)-c$ is separable for all elements $c \in \overline{\mathbb{F}}_{2}$.

Lemma 3.1. Let $c \in \overline{\mathbb{F}}_{q}$ be an element of the algebraic closure of $\mathbb{F}_{q}$. Then

$$
f(T)-c \text { is inseparable if and only if } q \neq 2 \text { and } c=1 .
$$

For an element $\beta \in \overline{\mathbb{F}}_{q}$ we have that $f(\beta)=1$ if and only if $\beta$ belongs to $\mathbb{F}_{q}$.

Proof. Just notice that the derivative of $f(T)$ satisfies $f^{\prime}(T)=\left(T^{q}-T\right)^{q-2}$.

Lemma 3.2. For an element $\beta \in \overline{\mathbb{F}}_{q}$ we have that $f(\beta)=0$ if and only if $\beta \in \mathbb{F}_{q^{2}} \backslash \mathbb{F}_{q}$.

Proof. Just notice that we have (see Rem. 2.1)

$$
f(T)=\left(T^{q^{2}}-T\right) /\left(T^{q}-T\right) .
$$

Now we consider the recursive equation for the tower $\mathcal{F}$ (see Eqn. (10)):

$$
f(Y)=\frac{1}{1-f(1 / X)} .
$$

We will show that if $X=\alpha$ belongs to $\mathbb{F}_{q^{3}} \backslash \mathbb{F}_{q}$ then all solutions $Y=\beta \in \overline{\mathbb{F}}_{q}$ of Eqn. (17) with $X=\alpha$ are such that $\beta \in \mathbb{F}_{q^{3}} \backslash \mathbb{F}_{q}$. The assertion that $\beta \notin \mathbb{F}_{q}$ follows directly from Eqn. (17) and the lemmas above.

Using Eqn. (16) we have:

$$
\frac{1}{1-f(T)}=\frac{T-T^{q}}{T^{q^{2}}-T^{q}}
$$


Lemma 3.3. For an element $\beta \in \overline{\mathbb{F}}_{q}$ we have that

$$
f(\beta)^{q}=\frac{1}{1-f(\beta)} \quad \text { if and only if } \quad \beta \in \mathbb{F}_{q^{3}} \backslash \mathbb{F}_{q} .
$$

Proof. Straightforward using Eqn. (16) and Eqn. (18).

Eqn. (17) can also be written as below:

$$
f\left(\frac{1}{X}\right)=1-\frac{1}{f(Y)} .
$$

Consider now a solution $(\alpha, \beta)$ of Eqn. (17) with $\alpha \in \mathbb{F}_{q^{3}} \backslash \mathbb{F}_{q}$. Then $1 / \alpha \in \mathbb{F}_{q^{3}} \backslash \mathbb{F}_{q}$. We have:

$$
f(\beta)=\frac{1}{1-f\left(\frac{1}{\alpha}\right)}=f\left(\frac{1}{\alpha}\right)^{q}=1-\frac{1}{f(\beta)^{q}} .
$$

In the last two equalities above we have used Lemma 3.3 and Eqn. (19), respectively. Hence we obtained that $f(\beta)^{q}=1 /(1-f(\beta))$; i.e., $\beta \in \mathbb{F}_{q^{3}} \backslash \mathbb{F}_{q}$.

We have thus proved the main result of this section:

Theorem 3.4. Let $\mathcal{F}=\left(F_{0}, F_{1}, \ldots\right)$ be the tower over $\mathbb{F}_{q^{3}}$ given recursively by Eqn. (17). Then the places $\left(x_{0}=\alpha\right)$ with $\alpha \in \mathbb{F}_{q^{3}} \backslash \mathbb{F}_{q}$ split completely in all extensions $F_{n} / F_{0}$. In particular the number of $\mathbb{F}_{q^{3}}$-rational places satisfies:

$$
N\left(F_{n}\right) \geq\left(q^{3}-q\right) \cdot\left[F_{n}: F_{0}\right] \quad \text { for all } n \in \mathbb{N} .
$$

\section{The extensions $K(x) / K(w)$ and $K(x) / K(u)$}

Throughout this section, $K$ is a field with $\mathbb{F}_{q^{2}} \subseteq K$. Let $K(x) / K$ be a rational function field over $K$. We will consider certain subfields $K(w) \subseteq K(x)$ and $K(u) \subseteq K(x)$ which are related to the recursive definition of the tower $\mathcal{F}$. Detailed information about ramification in $K(x) / K(w)$ and in $K(x) / K(u)$ will enable us to study in Sec. 5 and Sec. 6 the ramification behaviour in the tower $\mathcal{F}$.

As in Sec. 2 we consider the polynomial $f(T)=\left(T^{q}-T\right)^{q-1}+1 \in K[T]$, and we set

$$
w:=f(x)=\left(x^{q}-x\right)^{q-1}+1 \in K(x) .
$$

Lemma 4.1. (i) The extension $K(x) / K(w)$ is Galois of degree $q(q-1)$.

(ii) The place $(w=\infty)$ of $K(w)$ is totally ramified in $K(x) / K(w)$; the place above it is the place $(x=\infty)$. We have $d((x=\infty) \mid(w=\infty))=q^{2}-2 ;$ i.e., $(x=\infty) \mid(w=\infty)$ is weakly ramified.

(iii) Above the place $(w=1)$ there are the $q$ places $(x=\theta)$ of $K(x)$ with $\theta \in \mathbb{F}_{q}$, with ramification index $e((x=\theta) \mid(w=1))=q-1$.

(iv) All other places of $K(w)$ are unramified in $K(x) / K(w)$. 


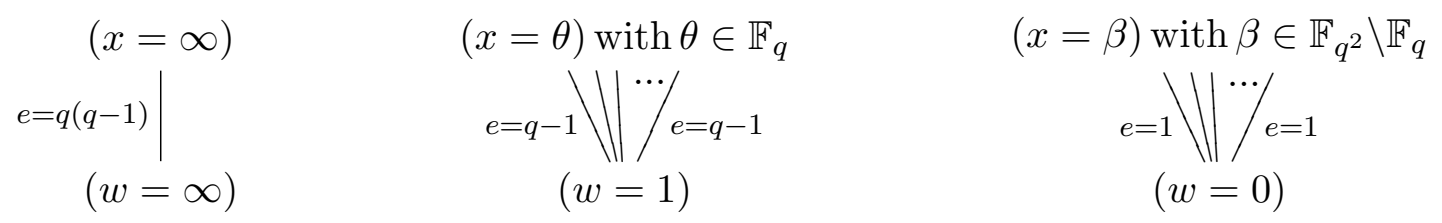

Figure 1: Ramification and splitting in $K(x) / K(w)$.

(v) The places above $(w=0)$ are exactly the places $(x=\beta)$ with $\beta \in \mathbb{F}_{q^{2}} \backslash \mathbb{F}_{q}$.

Proof. i) One checks easily that $K(w)$ is the fixed field of the following group $H$ of automorphisms of $K(x) / K$ :

$$
H:=\left\{\sigma \in \operatorname{Aut}(K(x) / K) \mid \sigma(x)=a x+b, a \in \mathbb{F}_{q}^{\times}, b \in \mathbb{F}_{q}\right\} .
$$

ii) It is clear from Eqn. (20) that $(x=\infty)$ is the only place of $K(x)$ lying above $(w=\infty)$, and that the ramification index is $e((x=\infty) \mid(w=\infty))=q(q-1)$. Since $K(x) / K(w)$ is Galois, it follows from ramification theory (cf. [16, Sec. III.8]) that $d((x=\infty) \mid(w=\infty)) \geq(q(q-1)-1)+(q-1)=q^{2}-2$. We will show below that equality holds; i.e., that $(x=\infty) \mid(w=\infty)$ is weakly ramified.

iii) This assertion is obvious from the equation $w-1=\left(x^{q}-x\right)^{q-1}$.

iv) It follows from above that the degree of the different $\operatorname{Diff}(K(x) / K(w))$ satisfies

$$
\begin{aligned}
\operatorname{deg} \operatorname{Diff}(K(x) / K(w)) & \geq d((x=\infty) \mid(w=\infty))+\sum_{\theta \in \mathbb{F}_{q}} d((x=\theta) \mid(w=1)) \\
& \geq\left(q^{2}-2\right)+q(q-2)=2\left(q^{2}-q-1\right) .
\end{aligned}
$$

On the other hand, by Hurwitz genus formula for $K(x) / K(w)$ we have

$$
\operatorname{deg} \operatorname{Diff}(K(x) / K(w))=-2+2[K(x): K(w)]=2\left(q^{2}-q-1\right) .
$$

Now the assertions iv) and ii) follow immediately.

v) Observing that (see Eqn. (16)) $w=f(x)=\left(x^{q^{2}}-x\right) /\left(x^{q}-x\right)$, we see that the places above $(w=0)$ are exactly the places $(x=\beta)$ with $\beta \in \mathbb{F}_{q^{2}} \backslash \mathbb{F}_{q}$.

Next we consider the subfield $K(u) \subseteq K(x)$ where $u$ is defined by

$$
u:=\frac{-x^{q(q-1)}}{\left(x^{q-1}-1\right)^{q-1}} .
$$

Lemma 4.2. (i) The extension $K(x) / K(u)$ is Galois of degree $q(q-1)$.

(ii) The place $(u=0)$ of $K(u)$ is totally ramified in $K(x) / K(u)$; the place above it is the place $(x=0)$. We have $d((x=0) \mid(u=0))=q^{2}-2$; i.e., $(x=0) \mid(u=0)$ is weakly ramified. 
(iii) Above the place $(u=\infty)$ lie exactly $q$ places $P$ of $K(x)$; namely the places $(x=\infty)$ and $(x=\alpha)$ with $\alpha \in \mathbb{F}_{q}^{\times}$. We have $e(P \mid(u=\infty))=q-1$.

(iv) No other place of $K(u)$ is ramified in $K(x)$.

(v) The places above $(u=1)$ are exactly the places $(x=\beta)$ with $\beta \in \mathbb{F}_{q^{2}} \backslash \mathbb{F}_{q}$.

$$
\begin{array}{ccc}
(x=0) & (x=\infty),(x=\alpha) \text { with } \alpha \in \mathbb{F}_{q}^{\times} & (x=\beta) \text { with } \beta \in \mathbb{F}_{q^{2}} \backslash \mathbb{F}_{q} \\
e=q(q-1) \mid & e=1 \backslash \|{ }_{e=1} \\
(u=0) & (u=\infty) & (u=1)
\end{array}
$$

Figure 2: Ramification and splitting in $K(x) / K(u)$.

Proof. Note that $u=1 /(1-f(1 / x))$ by Rem. 2.1 and therefore $f(1 / x)=(u-1) / u$. The result follows directly from Lemma 4.1 with the change of variables

$$
x \mapsto 1 / x \quad \text { and } \quad w \mapsto(u-1) / u .
$$

\section{The fields $F_{1}$ and $F_{2}$}

In this section we assume again that $\mathbb{F}_{q^{2}} \subseteq K$. We want to study ramification in the first two steps of the tower $\mathcal{F}$ over $K$. So we consider the fields $F_{0}=K\left(x_{0}\right), F_{1}=K\left(x_{0}, x_{1}\right)$ and $F_{2}=K\left(x_{0}, x_{1}, x_{2}\right)$ where

$$
\left(x_{1}^{q}-x_{1}\right)^{q-1}+1=\frac{-x_{0}^{q(q-1)}}{\left(x_{0}^{q-1}-1\right)^{q-1}} \quad \text { and } \quad\left(x_{2}^{q}-x_{2}\right)^{q-1}+1=\frac{-x_{1}^{q(q-1)}}{\left(x_{1}^{q-1}-1\right)^{q-1}} .
$$

Lemma 5.1. The extensions $F_{1} / K\left(x_{0}\right)$ and $F_{1} / K\left(x_{1}\right)$ are both Galois of degree $q(q-1)$.

Proof. We proved the assertion for $F_{1} / K\left(x_{0}\right)$ in Thm. 2.2. As in Eqn. (11) we set

$$
u_{0}:=\frac{-x_{0}^{q(q-1)}}{\left(x_{0}^{q-1}-1\right)^{q-1}} .
$$

The field $F_{1}$ is the compositum of $K\left(x_{0}\right)$ and $K\left(x_{1}\right)$ over $K\left(u_{0}\right)$ as in Figure 3. By Lemma 4.2 the extension $K\left(x_{0}\right) / K\left(u_{0}\right)$ is Galois, hence $F_{1} / K\left(x_{1}\right)$ is Galois as well.

Lemma 5.2. Let $\Omega:=\mathbb{F}_{q^{2}} \cup\{\infty\}$.

(i) For a place $P \in \mathbb{P}\left(F_{1}\right)$ the following are equivalent: 


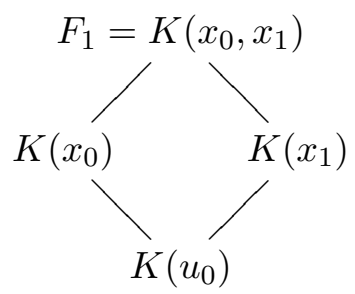

Figure 3: The extension $F_{1} / K\left(u_{0}\right)$

a) $\left.P\right|_{K\left(x_{0}\right)}=\left(x_{0}=\omega\right)$ for some $\omega \in \Omega$.

b) $\left.P\right|_{K\left(x_{1}\right)}=\left(x_{1}=\omega^{\prime}\right)$ for some $\omega^{\prime} \in \Omega$.

(ii) If a place $Q \in \mathbb{P}\left(F_{1}\right)$ does not lie above a place $\left(x_{0}=\omega\right)$ with $\omega \in \Omega$ then $Q$ is unramified over $K\left(x_{0}\right)$ and over $K\left(x_{1}\right)$.

(iii) The ramification indices of the places $\left(x_{0}=\omega\right)$ and $\left(x_{1}=\omega^{\prime}\right)$ with $\omega, \omega^{\prime} \in \Omega$ in the extensions $F_{1} / K\left(x_{0}\right)$ and $F_{1} / K\left(x_{1}\right)$ are as depicted in Figure 4. All places of $F_{1}$ are weakly ramified over $K\left(x_{0}\right)$ and over $K\left(x_{1}\right)$.
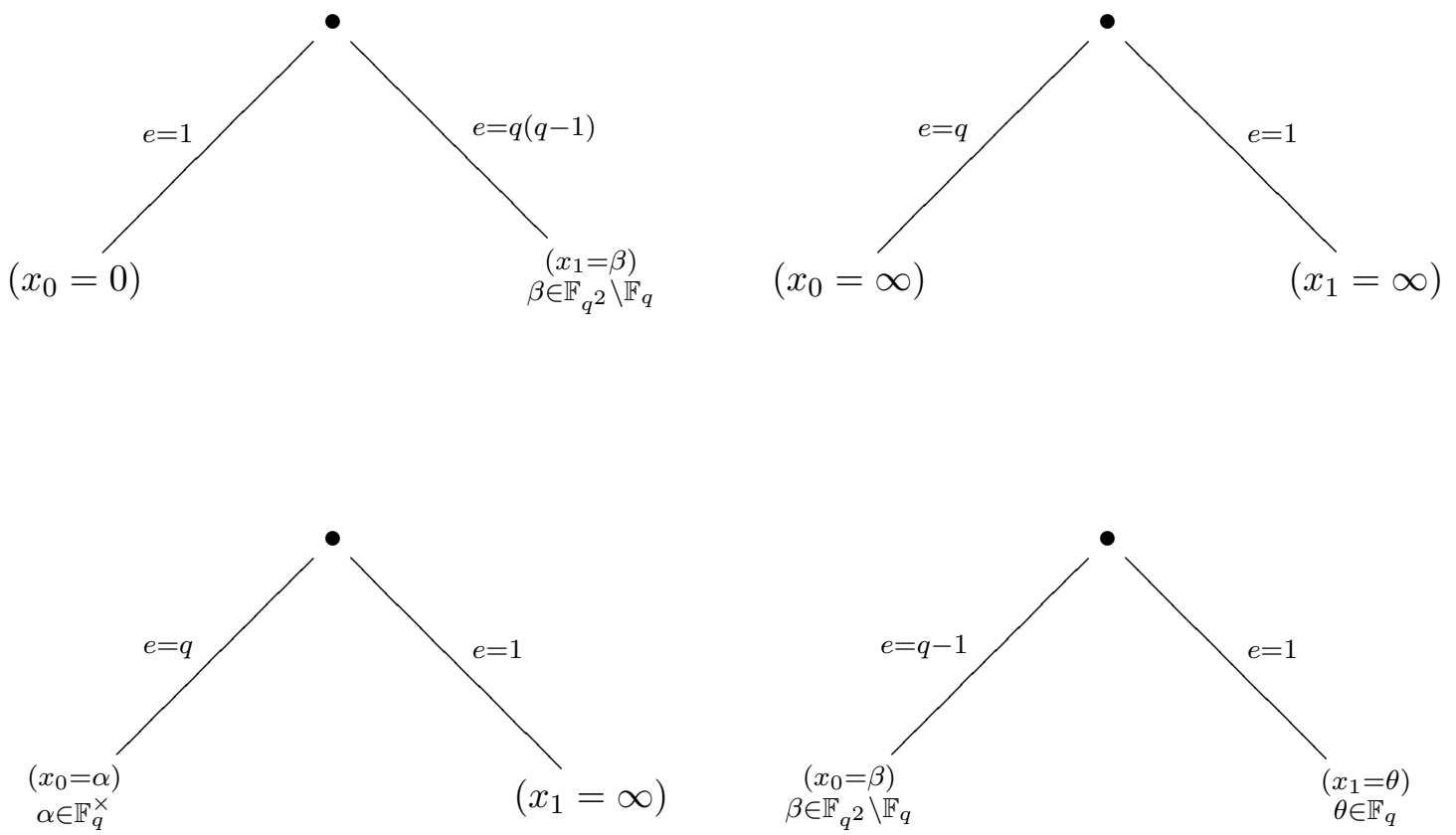

Figure 4: Ramification in $F_{1} / K\left(x_{0}\right)$ and in $F_{1} / K\left(x_{1}\right)$.

Proof. According to the notations in Sec. 4 we write $u_{0}:=-x_{0}^{q(q-1)} /\left(x_{0}^{q-1}-1\right)^{q-1}$ and $w_{1}:=\left(x_{1}^{q}-x_{1}\right)^{q-1}+1$. Hence $u_{0}=w_{1}$ by Eqn. (22). We consider the diagram of fields 
in Figure 3 where all extensions are Galois of degree $q(q-1)$. We have

$$
\begin{aligned}
& \left.P\right|_{K\left(x_{0}\right)}=\left(x_{0}=\omega\right) \text { for some } \omega \in \Omega \\
\Leftrightarrow & \left.\left.P\right|_{K\left(u_{0}\right)} \in\left\{\left(u_{0}=0\right),\left(u_{0}=1\right),\left(u_{0}=\infty\right)\right\} \text { (by Lemma } 4.2\right) \\
\Leftrightarrow & \left.P\right|_{K\left(x_{1}\right)}=\left(x_{1}=\omega^{\prime}\right) \text { for some } \omega^{\prime} \in \Omega(\text { by Lemma } 4.1) .
\end{aligned}
$$

By Lemma 4.1 and Lemma 4.2 we know that only the places $\left(u_{0}=0\right),\left(u_{0}=1\right)$ and $\left(u_{0}=\infty\right)$ are ramified in $K\left(x_{0}\right) / K\left(u_{0}\right)$ or in $K\left(x_{1}\right) / K\left(u_{0}\right)$. We will consider here only the case $\left(u_{0}=\infty\right)$; the other two cases are similar (even easier). Denote by $Q$ a place of $F_{1}$ above $\left(u_{0}=\infty\right)$. The situation is depicted in Figure 5. It follows

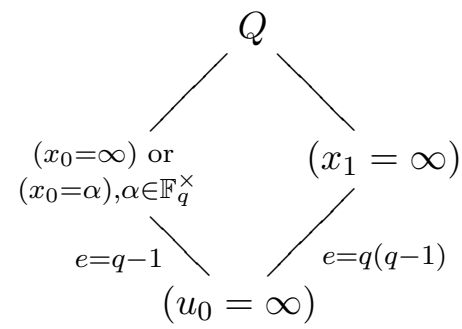

Figure 5: Ramification in $F_{1} / K\left(u_{0}\right)$

from Abhyankar's Lemma (see [16, Prop. III.8.9]) that $Q$ is unramified over $K\left(x_{1}\right)$ and that the ramification index of $Q$ over $K\left(x_{0}\right)$ is $e=q$. Since $\left(x_{1}=\infty\right) \mid\left(u_{0}=\infty\right)$ is weakly ramified by Lemma 4.1, it follows from the transitivity of different exponents in $F_{1} \supseteq K\left(x_{0}\right) \supseteq K\left(u_{0}\right)$ that $Q$ is weakly ramified over $K\left(x_{0}\right)$.

Lemma 5.3. The extensions $F_{2} / K\left(x_{0}, x_{1}\right)$ and $F_{2} / K\left(x_{1}, x_{2}\right)$ are Galois extensions of degree $q$. All places that are ramified in $F_{2} / K\left(x_{0}, x_{1}\right)$ or in $F_{2} / K\left(x_{1}, x_{2}\right)$ are totally and weakly ramified.

Proof. The field $F_{2}$ is the compositum of $K\left(x_{0}, x_{1}\right)$ and $K\left(x_{1}, x_{2}\right)$ over $K\left(x_{1}\right)$. Since the extensions $K\left(x_{0}, x_{1}\right) / K\left(x_{1}\right)$ and $K\left(x_{1}, x_{2}\right) / K\left(x_{1}\right)$ are Galois by Lemma 5.1, it is clear that $F_{2} / K\left(x_{0}, x_{1}\right)$ and $F_{2} / K\left(x_{1}, x_{2}\right)$ are Galois. The assertion about the degrees follows from Lemma 2.7. Now we consider a place $Q \in \mathbb{P}\left(F_{2}\right)$ which is ramified in $F_{2} / K\left(x_{1}, x_{2}\right)$. Then the place $P:=\left.Q\right|_{K\left(x_{0}, x_{1}\right)}$ is ramified over $K\left(x_{1}\right)$ and therefore $\left.Q\right|_{K\left(x_{1}\right)}=\left(x_{1}=\beta\right)$ with some $\beta \in \mathbb{F}_{q^{2}} \backslash \mathbb{F}_{q}$, by Lemma 5.2. So we have the situation depicted in Figure 6, where $R$ denotes the restriction of $Q$ to $K\left(x_{1}, x_{2}\right)$.

As in the proof of Lemma 5.2, we use Abhyankar's lemma to get that $e(Q \mid R)=q$, and the transitivity of different exponents to get that $d(Q \mid R)=2 \cdot(q-1)$.

Now if $Q$ is a place of $F_{2}$ which is ramified over $F_{1}$, then one also concludes (and it is simpler) that it is totally and weakly ramified over $F_{1}$.

Remark 5.4. It is clear that all statements in this section remain valid when the fields $K\left(x_{0}\right), K\left(x_{0}, x_{1}\right)$ and $K\left(x_{0}, x_{1}, x_{2}\right)$ are replaced by the fields $K\left(x_{n}\right), K\left(x_{n}, x_{n+1}\right)$ and $K\left(x_{n}, x_{n+1}, x_{n+2}\right)$, respectively. 

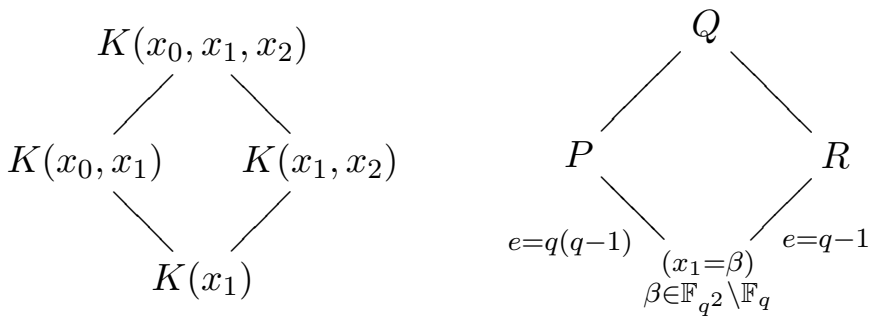

Figure 6:

\section{The genus of $F_{n}$}

In order to estimate the limit $\lambda(\mathcal{F})$ of the tower $\mathcal{F}$ over $\mathbb{F}_{q^{3}}$ we need an upper bound for the genus of the $n$-th function field $F_{n}$; therefore one has to study ramification in the extension $F_{n} / F_{0}$. Without changing the ramification behaviour (i.e., ramification index and different exponent) and the genus, we can extend the constant field such that it contains $\mathbb{F}_{q^{2}}$. So we assume in this section that $\mathbb{F}_{q^{2}} \subseteq K$ and denote $\operatorname{char}(K)=p$.

A place $P \in \mathbb{P}\left(F_{0}\right)$ is said to be ramified in the tower $\mathcal{F}$ if $P$ is ramified in $F_{m} / F_{0}$ for some $m \geq 1$, and the ramification locus $V\left(\mathcal{F} / F_{0}\right)$ is defined as

$$
V\left(\mathcal{F} / F_{0}\right):=\left\{P \in \mathbb{P}\left(F_{0}\right) \mid P \text { is ramified in } \mathcal{F}\right\} .
$$

Lemma 6.1. The ramification locus of $\mathcal{F}$ over $F_{0}$ satisfies

$$
V\left(\mathcal{F} / F_{0}\right) \subseteq\left\{\left(x_{0}=\omega\right) \mid \omega \in \mathbb{F}_{q^{2}} \text { or } \omega=\infty\right\} .
$$

Proof. Assume that a place $Q \in \mathbb{P}\left(F_{n}\right)$ is ramified in $F_{n+1} / F_{n}$. Then the restriction $\left.Q\right|_{K\left(x_{n}\right)}$ ramifies in the extension $K\left(x_{n}, x_{n+1}\right) / K\left(x_{n}\right)$. We conclude from Lemma 5.2 ii) that $\left.Q\right|_{K\left(x_{n}\right)}=\left(x_{n}=\omega^{\prime}\right)$ with $\omega^{\prime} \in \mathbb{F}_{q^{2}} \cup\{\infty\}$. By induction it follows from Lemma 5.2 i) that $\left.Q\right|_{F_{0}}=\left(x_{0}=\omega\right)$ with $\omega \in \mathbb{F}_{q^{2}} \cup\{\infty\}$. This proves the lemma. We remark that in fact $V\left(\mathcal{F} / F_{0}\right)=\left\{\left(x_{0}=\omega\right) \mid \omega \in \mathbb{F}_{q^{2}}\right.$ or $\left.\omega=\infty\right\}$ but we do not need this here.

In the proof of Lemma 6.3 below, the following result is crucial:

Lemma 6.2. Consider an extension $E / F$ of function fields over $K$ such that $E=E_{1} \cdot E_{2}$ is the composite field of two intermediate fields $F \subseteq E_{i} \subseteq E, i=1,2$ and the extensions $E_{1} / F$ and $E_{2} / F$ are Galois p-extensions. Let $Q$ be a place of $E$, and let $Q_{i}:=\left.Q\right|_{E_{i}}$ and $P:=\left.Q\right|_{F}$ be the restrictions of $Q$. Suppose that $Q_{1} \mid P$ and $Q_{2} \mid P$ are weakly ramified. Then $Q \mid Q_{1}$ and $Q \mid Q_{2}$ are also weakly ramified.

Proof. See [10, Prop. 1.10] and also [9, Lemma 1].

A Galois extension $E / F$ is weakly ramified if all places are weakly ramified in $E / F$.

Lemma 6.3. Let $n \geq 1$. Then the extension $F_{n+1} / F_{n}$ is weakly ramified. 
Proof. For $0 \leq i \leq j \leq n+1$ we define the subfield $E_{i, j} \subseteq F_{n+1}$ by

$$
E_{i, j}:=K\left(x_{i}, x_{i+1}, \ldots, x_{j}\right) .
$$

The extensions $E_{i, i+2} / E_{i, i+1}$ and $E_{i, i+2} / E_{i+1, i+2}$ are weakly ramified Galois $p$-extensions by Lemma 5.3 (see Figure 7). By induction it follows for all $j \geq i+2$ that $E_{i, j} / E_{i, j-1}$ and $E_{i, j} / E_{i+1, j}$ are weakly ramified Galois $p$-extensions (using Lemma 6.2). Since $F_{n}=E_{0, n}$ and $F_{n+1}=E_{0, n+1}$, the assertion of Lemma 6.3 follows.

Lemma 6.4. Let $E_{1} / F$ be a Galois extension of function fields over $K$ and let $E / E_{1}$ be a finite and separable extension. Let $Q$ be a place of the field $E$ and denote by $P_{1}$ and $P$ the restrictions of $Q$ to $E_{1}$ and $F$, respectively. Suppose that we have:

(i) $e\left(Q \mid P_{1}\right)$ is a power of $p=\operatorname{char}(K)$ and $d\left(Q \mid P_{1}\right)=2 e\left(Q \mid P_{1}\right)-2$.

(ii) The place $P_{1}$ is weakly ramified over $P$.

Then the different exponent $d(Q \mid P)$ satisfies

$$
d(Q \mid P)=\left(e_{0} e_{1}-1\right)+\left(e_{1}-1\right)<e(Q \mid P) \cdot\left(1+\frac{1}{e_{0}}\right),
$$

where $e(Q \mid P)=e_{0} e_{1}$ with $\left(p, e_{0}\right)=1$ and $e_{1}$ is a p-power.

Proof. Straightforward, using transitivity of different exponents.

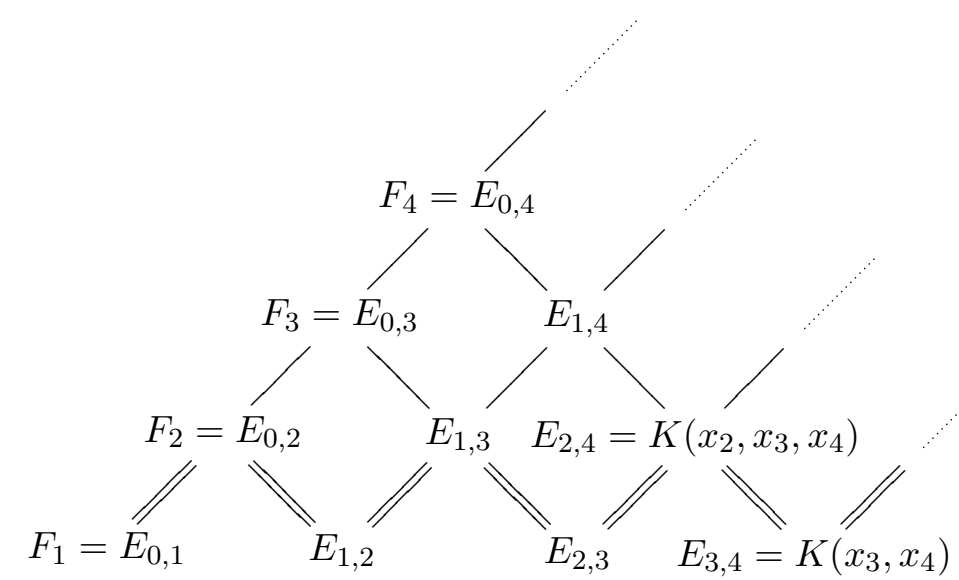

Figure 7: Double lines denote weakly ramified Galois p-extensions

Theorem 6.5. The genus of the $n$-th function field of the tower $\mathcal{F}=\left(F_{0}, F_{1}, F_{2}, \ldots\right)$ defined by Eqn. (8), satisfies

$$
g\left(F_{n}\right) \leq \frac{q^{2}+2 q}{2} \cdot\left[F_{n}: F_{0}\right]
$$


Proof. Let $n \geq 1$. First we observe that for a place $Q \in \mathbb{P}\left(F_{n}\right)$ and the restriction $P_{1}:=\left.Q\right|_{F_{1}}$ of $Q$ to $F_{1}$ we have that

$$
e\left(Q \mid P_{1}\right) \text { is a } p \text {-power and } d\left(Q \mid P_{1}\right)=2 e\left(Q \mid P_{1}\right)-2 .
$$

This follows from Lemma 6.3 and repeated applications of Lemma 6.4.

Now we consider the places $P \in \mathbb{P}\left(F_{0}\right)$ which are in the ramification locus $V\left(\mathcal{F} / F_{0}\right)$. According to item (iii) of Lemma 5.2 we distinguish 2 cases:

Case 1: $P=\left(x_{0}=\theta\right)$ with $\theta \in \mathbb{F}_{q}$ or $P=\left(x_{0}=\infty\right)$.

By Lemma 5.2 and Lemma 6.4 we obtain

$$
\sum_{\substack{Q \in \mathbb{P}\left(F_{n}\right) \\ Q \mid P}} d(Q \mid P) \cdot \operatorname{deg} Q<\sum_{\substack{Q \in \mathbb{P}\left(F_{n}\right) \\ Q \mid P}} 2 e(Q \mid P) \cdot \operatorname{deg} Q=2\left[F_{n}: F_{0}\right] .
$$

Case 2: $P=\left(x_{0}=\beta\right)$ with $\beta \in \mathbb{F}_{q^{2}} \backslash \mathbb{F}_{q}$.

In this case, Lemma 5.2 and Lemma 6.4 yield

$$
\sum_{\substack{Q \in \mathbb{P}\left(F_{n}\right) \\ Q \mid P}} d(Q \mid P) \cdot \operatorname{deg} Q<\sum_{\substack{Q \in \mathbb{P}\left(F_{n}\right) \\ Q \mid P}}\left(1+\frac{1}{q-1}\right) e(Q \mid P) \cdot \operatorname{deg} Q=\frac{q}{q-1}\left[F_{n}: F_{0}\right] .
$$

There are $q+1$ places $P \in \mathbb{P}\left(F_{0}\right)$ as in Case 1 , and $q^{2}-q$ places as in Case 2. By Hurwitz genus formula for the extension $F_{n} / F_{0}$ we obtain

$$
\begin{aligned}
2 g\left(F_{n}\right) & \leq-2\left[F_{n}: F_{0}\right]+(q+1) \cdot 2\left[F_{n}: F_{0}\right]+\left(q^{2}-q\right) \cdot \frac{q}{q-1}\left[F_{n}: F_{0}\right] \\
& =\left(q^{2}+2 q\right)\left[F_{n}: F_{0}\right] .
\end{aligned}
$$

\section{The limit of the tower over $K=\mathbb{F}_{\ell}$ with $\ell=q^{3}$}

Putting together the results of the previous sections we obtain our main result:

Theorem 7.1. Let $K=\mathbb{F}_{\ell}$ with $\ell=q^{3}$, and let $\mathcal{F}=\left(F_{0}, F_{1}, F_{2}, \ldots\right)$ be the tower over $K$ which is recursively defined by $F_{0}=K\left(x_{0}\right)$ and $F_{n+1}=F_{n}\left(x_{n+1}\right)$, where

$$
\left(x_{n+1}^{q}-x_{n+1}\right)^{q-1}+1=\frac{-x_{n}^{q(q-1)}}{\left(x_{n}^{q-1}-1\right)^{q-1}} \quad \text { for all } n \geq 0 .
$$

Then the limit $\lambda(\mathcal{F})=\lim _{n \rightarrow \infty} N\left(F_{n}\right) / g\left(F_{n}\right)$ satisfies

$$
\lambda(\mathcal{F}) \geq 2\left(q^{2}-1\right) /(q+2) .
$$


Proof. By Thm. 3.4 and Thm. 6.5 we have

$$
N\left(F_{n}\right) \geq\left(q^{3}-q\right) \cdot\left[F_{n}: F_{0}\right] \text { and } g\left(F_{n}\right) \leq \frac{q^{2}+2 q}{2} \cdot\left[F_{n}: F_{0}\right] .
$$

Hence

$$
\frac{N\left(F_{n}\right)}{g\left(F_{n}\right)} \geq \frac{\left(q^{3}-q\right) \cdot 2}{q^{2}+2 q}=\frac{2\left(q^{2}-1\right)}{q+2} \text { for all } n \geq 0
$$

\section{Remarks}

We finish this paper with a few remarks.

Remark 8.1. Our tower $\mathcal{F}=\left(F_{0}, F_{1}, F_{2}, \ldots\right)$ over $K=\mathbb{F}_{q^{3}}$ bears remarkable analogy to the tower $\mathcal{H}=\left(H_{0}, H_{1}, H_{2}, \ldots\right)$ over the quadratic field $K=\mathbb{F}_{q^{2}}$ which is defined recursively by the equation

$$
u_{i+1}^{q}+u_{i+1}=\frac{u_{i}^{q}}{u_{i}^{q-1}+1}
$$

and which attains the Drinfel'd-Vlădut bound (1). The analogies between $\mathcal{H}$ and $\mathcal{F}$ become even more evident if we substitute $u_{i}=\xi y_{i}$ with $\xi^{q-1}=-1$; then the above equation becomes $y_{i+1}^{q}-y_{i+1}=-y_{i}^{q} /\left(y_{i}^{q-1}-1\right)$. We now compare some features of the towers $\mathcal{F}$ over $\mathbb{F}_{q^{3}}$ and $\mathcal{H}$ over $\mathbb{F}_{q^{2}}$, see [8].

1) The tower $\mathcal{H}=\left(H_{0}, H_{1}, H_{2}, \ldots\right)$ is defined recursively over the field $K=\mathbb{F}_{q^{2}}$ by $H_{0}=K\left(y_{0}\right)$ and $H_{i+1}=H_{i}\left(y_{i+1}\right)$, where

$$
y_{i+1}^{q}-y_{i+1}=\frac{-y_{i}^{q}}{y_{i}^{q-1}-1} \quad \text { for all } i \geq 0 .
$$

2) Setting $h(T):=T^{q}-T$, Eqn. (25) can be written as

$$
h\left(y_{i+1}\right)=\frac{1}{h\left(1 / y_{i}\right)} .
$$

3) The extensions $H_{i+1} / H_{i}$ (for $i \geq 0$ ) are weakly ramified Galois extensions of degree $\left[H_{i+1}: H_{i}\right]=q$.

4) The ramification locus of $\mathcal{H}$ over $H_{0}$ is

$$
V\left(\mathcal{H} / H_{0}\right)=\left\{\left(y_{0}=\omega\right) \mid \omega \in \mathbb{F}_{q} \cup\{\infty\}\right\} .
$$

5) The places $\left(y_{0}=\alpha\right)$ with $\alpha \in \mathbb{F}_{q^{2}} \backslash \mathbb{F}_{q}$ are completely splitting in the extensions $H_{n} / H_{0}$, for all $n \geq 0$.

The analogous properties of the tower $\mathcal{F}$ are: 
$\left.1^{*}\right)$ The tower $\mathcal{F}=\left(F_{0}, F_{1}, F_{2}, \ldots\right)$ is defined recursively over the field $K=\mathbb{F}_{q^{3}}$ by $F_{0}=K\left(x_{0}\right)$ and $F_{i+1}=F_{i}\left(x_{i+1}\right)$, where

$$
\left(x_{i+1}^{q}-x_{i+1}\right)^{q-1}+1=\frac{-x_{i}^{q(q-1)}}{\left(x_{i}^{q-1}-1\right)^{q-1}} \quad \text { for all } i \geq 0 .
$$

$\left.2^{*}\right)$ Setting $f(T):=\left(T^{q}-T\right)^{q-1}+1$, Eqn. (27) can be written as

$$
f\left(x_{i+1}\right)=\frac{1}{1-f\left(1 / x_{i}\right)} .
$$

$3^{*}$ ) The extensions $F_{i+1} / F_{i}$ (for $i \geq 1$ ) are weakly ramified Galois extensions of degree $\left[F_{i+1}: F_{i}\right]=q$.

$\left.4^{*}\right)$ The ramification locus of $\mathcal{F}$ over $F_{0}$ is

$$
V\left(\mathcal{F} / F_{0}\right)=\left\{\left(x_{0}=\omega\right) \mid \omega \in \mathbb{F}_{q^{2}} \cup\{\infty\}\right\} .
$$

$\left.5^{*}\right)$ The places $\left(x_{0}=\alpha\right)$ with $\alpha \in \mathbb{F}_{q^{3}} \backslash \mathbb{F}_{q}$ are completely splitting in the extensions $F_{n} / F_{0}$, for all $n \geq 0$.

We also note that the polynomials $h(T)$ and $f(T)$ in Eqn. (26) and Eqn. (28) are defined in a very similar manner:

6) The polynomial $h(T) \in \mathbb{F}_{q}[T]$ generates the fixed field of $K(T)$ under the group of automorphisms

$$
G=\left\{\sigma: K(T) \rightarrow K(T) \mid \sigma(T)=T+b \text { with } b \in \mathbb{F}_{q}\right\} .
$$

$\left.6^{*}\right)$ The polynomial $f(T) \in \mathbb{F}_{q}[T]$ generates the fixed field of $K(T)$ under the group of automorphisms

$$
G^{*}=\left\{\sigma: K(T) \rightarrow K(T) \mid \sigma(T)=a T+b \text { with } a \in \mathbb{F}_{q}^{\times} \text {and } b \in \mathbb{F}_{q}\right\} .
$$

Another interesting observation is that the generators $x_{i}$ of the tower $\mathcal{F}$ satisfy

$$
x_{i+2}^{q}-x_{i+2}=\frac{-x_{i}^{q}}{\left(x_{i}^{q-1}-1\right)\left(x_{i+1}^{q-1}-1\right)}
$$

for all $i \geq 0$ (with an appropriate choice of the roots $x_{i+1}, x_{i+2}$ of Eqn. (27); see Lemma 2.7). Compare with Eqn. (25).

Remark 8.2. The first explicit tower over a field with cubic cardinality $\ell=q^{3}$ which attains the Zink bound (Inequality (2)) was found by van der Geer-van der Vlugt [12]. It is a tower over the field $\mathbb{F}_{p^{3}}$ with $p=2$, recursively defined by the equation

$$
x_{i+1}^{2}+x_{i+1}=x_{i}+1+\frac{1}{x_{i}} .
$$

This is the special case $q=2$ of Eqn. (27) (after the change of variables $x_{i} \rightarrow x_{i}+1$ ). 
Remark 8.3. Again we consider the tower $\mathcal{F}=\left(F_{0}, F_{1}, F_{2}, \ldots\right)$ over $K=\mathbb{F}_{q^{3}}$. We set

$$
v_{i}:=-\frac{1}{x_{i}^{q-1}-1} \quad \text { for all } \quad i \geq 0
$$

It follows by straightforward calculations from Eqn. (27) that

$$
\frac{1-v_{i+1}}{v_{i+1}^{q}}=\frac{v_{i}^{q}+v_{i}-1}{v_{i}}, \quad \text { for all } i \geq 0 .
$$

This means that $\mathcal{F}$ contains as a subtower the tower $\mathcal{E}=\left(E_{0}, E_{1}, E_{2}, \ldots\right)$ (see [3]) with $E_{0}=K\left(v_{0}\right)$ and $E_{i+1}=E_{i}\left(v_{i+1}\right)$, where $v_{i+1}$ satisfies Eqn. (32) over $E_{i}$. Since the limit of a subtower is at least as big as the limit of the tower itself (see [8]), we obtain that

$$
\lambda(\mathcal{E}) \geq \lambda(\mathcal{F}) \geq \frac{2\left(q^{2}-1\right)}{q+2} .
$$

This gives another (in fact, much simpler) proof of the main result of [3].

Here is another striking analogy between $\mathcal{F}$ and $\mathcal{H}$; again we consider the tower $\mathcal{H}=\left(H_{0}, H_{1}, H_{2}, \ldots\right)$ over $K=\mathbb{F}_{q^{2}}$ given recursively by

$$
u_{i+1}^{q}+u_{i+1}=\frac{u_{i}^{q}}{u_{i}^{q-1}+1} .
$$

Performing the analogous change of variables as in Eqn. (31); i.e., setting

$$
w_{i}:=-\frac{1}{u_{i}^{q-1}+1} \quad \text { for all } i \geq 0,
$$

it follows by straightforward calculations from Eqn. (33) that

$$
\frac{w_{i+1}+1}{w_{i+1}^{q}}=\frac{w_{i}^{q}+1}{w_{i}}, \quad \text { for all } i \geq 0 .
$$

The subtower $\mathcal{G}$ of $\mathcal{H}$ given recursively by Eqn. (34) was studied in [2].

Remark 8.4. We end up this paper with a closer look on the relations between the towers $\mathcal{F}$ and $\mathcal{E}$ given by Eqns. (27) and (32), respectively. One can show that $F_{1} / E_{1}$ is a Galois extension of degree $(q-1)^{2}$ with group $\mathbb{F}_{q}^{\times} \times \mathbb{F}_{q}^{\times}$; in fact the automorphisms of $F_{1}=\mathbb{F}_{q^{3}}\left(x_{0}, x_{1}\right)$ over the subfield $E_{1}=\mathbb{F}_{q^{3}}\left(v_{0}, v_{1}\right)$ are given by:

$$
x_{0} \mapsto a x_{0} \text { and } x_{1} \mapsto b x_{1} \text {, with } a, b \in \mathbb{F}_{q}^{\times} .
$$

Moreover the $n$-th field $F_{n}$ of the tower $\mathcal{F}$ is the compositum with $F_{1}$ of the $n$-th field $E_{n}$ of the tower $\mathcal{E}$; i.e., we have

$$
F_{n}=E_{n} \cdot F_{1}, \quad \text { for all } n \geq 1 .
$$


The assertions above follow from Eqns. (31) and (29). We note however that for $q \neq 2$ the towers $\mathcal{F}$ and $\mathcal{E}$ are not $K$-isomorphic; i.e., there is no $K$-isomorphism

$$
\sigma: \bigcup_{i=0}^{\infty} F_{i} \longrightarrow \bigcup_{j=0}^{\infty} E_{j} .
$$

In order to prove this we assume that such an isomorphism $\sigma$ exists. Then we find integers $n \geq 2$ and $s \geq 2$ such that

$$
\sigma\left(F_{1}\right) \subseteq E_{n} \subseteq E_{n+1} \subseteq \sigma\left(F_{s}\right) .
$$

In the extension $\sigma\left(F_{s}\right) / \sigma\left(F_{1}\right)$ there occurs only wild ramification by Theorem 2.2, but in the extension $E_{n+1} / E_{n}$ there is also some tame ramification with ramification index $e=q-1$, cf. [3], p.177, Fig.1.

\section{Acknowledgment}

We would like to thank Y. Ihara for his interest in and helpful discussions about splitting places in the tower $\mathcal{E}$, cf. [14].

\section{References}

[1] A. Bassa, H. Stichtenoth, A simplified proof for the limit of a tower over a cubic finite field, J. Number Theory 123, 2007, 154-169.

[2] J. Bezerra, A. Garcia, A tower with non-Galois steps which attains the DrinfeldVladut bound, J. Number Theory 106, 2004, 142-154.

[3] J. Bezerra, A. Garcia, H. Stichtenoth, An explicit tower of function fields over cubic finite fields and Zink's lower bound, J. Reine Angew. Math. 589, 2005, 159-199.

[4] V. G. Drinfel'd, S. G. Vlăduţ, The number of points of an algebraic curve, Func. Anal. 17, 1983, 53-54.

[5] N. Elkies, Explicit modular towers, Proceedings of the 35th Annual Allerton Conference on Communication, Control and Computing (eds. T. Basar et al.) Urbana IL, 1997, 23-32.

[6] N. Elkies, Explicit towers of Drinfeld modular curves, European Congress of Math. (Barcelona, 2000) Vol. II, 189-198, Progr. Math., 202, Birkhäuser, Basel, 2001.

[7] A. Garcia, H. Stichtenoth, A tower of Artin-Schreier extensions of function fields attaining the Drinfeld-Vladut bound, Inventiones Math. 121, 1995, 211-222.

[8] A. Garcia, H. Stichtenoth, On the asymptotic behaviour of some towers of functions fields over finite fields, J. Number Theory 61, 1996, 248-273.

[9] A. Garcia, H. Stichtenoth, Some Artin-Schreier towers are easy, Mosc. Math. J. 5, 2005, 767-774. 
[10] A. Garcia, H. Stichtenoth, On the Galois closure of towers, Recent Trends in Coding Theory and its Applications (W. Li, ed.), to appear.

[11] A. Garcia, H. Stichtenoth, Explicit towers of function fields over finite fields, Topics in geometry, coding theory and cryptography, 1-58, Algebr. Appl., 6, Springer, Dordrecht, 2007.

[12] G. van der Geer, M. van der Vlugt, An asymptotically good tower of curves over the field with eight elements, Bull. London Math. Soc. 34, 2002, 291-300.

[13] Y. Ihara, Some remarks on the number of rational points of algebraic curves over finite fields, J. Fac. Sci. Univ. Tokyo Sect. IA Math. 28, 1981, 721-724.

[14] Y. Ihara, Some Remarks on the BGS Tower over Finite Cubic Fields, Proceedings of the conference "Arithmetic Geometry, Related Area and Applications" held at Chuo University, April 2006, 127-131.

[15] J.-P. Serre, Sur le nombre des points rationnels d'une courbe algébrique sur un corps fini, C. R. Acad. Sci. Paris 296, 1983, 397-402.

[16] H. Stichtenoth, Algebraic function fields and codes, Springer Verlag, Berlin, 1993.

[17] M. A. Tsfasman, S. G. Vlăduţ, T. Zink, Modular curves, Shimura curves, and Goppa codes, better than the Varshamov-Gilbert bound, Math. Nachr. 109, 1982, 21-28.

[18] T. Zink, Degeneration of Shimura surfaces and a problem in coding theory, in Fundamentals of Computation Theory (L. Budach, ed.), Lecture Notes in Computer Science, Vol. 199, Springer Verlag, Berlin, 1985, 503-511. 